\title{
Papel de las alteraciones del sueño durante la gestación en la programación del feto para el desarrollo de obesidad y enfermedades crónicas degenerativas
}

\author{
María del Rosario Ayala-Moreno, ${ }^{1 *}$ Rafael Velázquez-Martínez, ${ }^{1}$ Montserrat Melgarejo-Gutiérrez, ${ }^{2}$ \\ Claudia González-Méndez, ${ }^{1}$ Erika Estrada-Ramírez ${ }^{1}$ y Arely Vergara-Castañeda ${ }^{1}$ \\ 'Universidad La Salle, Facultad de Ciencias Químicas, Grupo de Investigación en Ciencias Básicas y Clínicas de la Salud, Ciudad de México; \\ ${ }^{2}$ Universidad Veracruzana, Campus Xalapa, Facultad de Medicina, Jalapa, Veracruz. México
}

\section{Resumen}

En el tercer trimestre del embarazo son frecuentes las alteraciones del sueño, las cuales generan cambios en la secreción de melatonina en mujeres gestantes que duermen menos de ocho horas o presentan alteraciones de sueño, promoviendo diversos cambios fisiológicos en la madre, que a su vez derivan en bajo peso al nacimiento (BPN) en el producto. El bajo peso al nacimiento está asociado con un fenómeno conocido como "programación metabólica", en la que el feto es sometido a estrés que tiene como resultado alteraciones metabólicas irreversibles que lo predisponen al desarrollo de obesidad en la edad adulta.

PALABRAS CLAVE: Alteraciones del sueño. Melatonina. Programación metabólica. Obesidad. Enfermedades metabólicas.

\begin{abstract}
Sleep disturbances are common in the third trimester of pregnancy and generate changes in the secretion of melatonin in pregnant women who sleep less than eight hours or have sleep disturbances, which promote various physiological changes in the mother that in turn result in low birth weight (LBW) in the fetus. LBW is associated with a phenomenon known as "metabolic programming," in which the fetus is subjected to a stressful situation that results in irreversible metabolic alterations that predispose it to the development of obesity in adulthood.
\end{abstract}

KEY WORDS: Sleep disturbances. Melatonin. Metabolic programming. Obesity. Metabolic diseases.

\section{Introducción}

El sueño como proceso fisiológico y sus alteraciones es un tema que ha cobrado importancia en los últimos años, por su impacto en el estado nutricional ${ }^{1-3} y$ la presencia de enfermedades crónicas degenerativas, ${ }^{4,5}$ que aumentan el riesgo de enfermedad cardiovascular. ${ }^{1}$
El sueño no es solamente la ausencia de vigilia, es un estado durante el cual el cerebro realiza una serie de actividades fisiológicas muy especializadas. Es un proceso en el que se mantiene activo el metabolismo, la restauración de tejidos, la consolidación de la memoria y el balance homeostático general. ${ }^{6}$ Por ello, cualquier alteración de este proceso que conlleve disminución del tiempo total de
Correspondencia:

*María del Rosario Ayala-Moreno

E-mail: rosario.ayala@lasalle.mx
Fecha de recepción: 18-10-2018

Fecha de aceptación: 29-10-2018

DOI: 10.24875/GMM.18004759
Gac Med Mex. 2019;155:423-427

Disponible en PubMed www.gacetamedicademexico.com 
sueño (TTS) puede repercutir en el mantenimiento de las actividades fisiológicas, y afectar seriamente el estado de salud físico, mental e interacción social del individuo.? El impacto negativo de la reducción en el TTS se ha estudiado en individuos adultos, jóvenes y población pediátrica, sin embargo, las condiciones fisiológicas del embarazo y lactancia han sido menos abordadas, aun cuando se ha observado que las afecciones del estado nutricional y metabólico en etapas tempranas del desarrollo favorecen el fenómeno conocido como "programación metabólica" u "origen temprano de las enfermedades metabólicas", procesos mediante los cuales el organismo es protegido del ambiente estresante al que es sometido, pero que tienen repercusiones a largo plazo al ser irreversibles y asociarse estrechamente con el aumento de la prevalencia de obesidad y enfermedades metabólicas en el sujeto adulto. ${ }^{8}$

En el embarazo, la secreción de las hormonas maternas es afectada por el ciclo de luz-oscuridad, entre ellas la melatonina, hormona estimulante de la tiroides, hormona de crecimiento y cortisol, por lo que se ha sugerido que los procesos metabólicos que estas hormonas regulan pueden ser seriamente afectados ${ }^{9}$ alterando la fisiología fetal.

La importancia de la reducción del TTS sobre la programación metabólica está siendo investigada, incluso, se ha propuesto que el tratamiento con melatonina puede generar un fenómeno de "reprogramación metabólica", el cual puede modificar o atenuar el daño generado por la programación temprana. ${ }^{10}$

\section{Generalidades del sueño}

El sueño es un proceso activo y regulado, esencial para conservar la salud y el bienestar. ${ }^{9}$ Este se puede dividir en dos etapas: el sueño de ondas lentas y el sueño de movimientos oculares rápidos o sueño REM (rapid eye movement). El primero se denomina de esta forma porque durante esta etapa el registro electroencefalográfico reporta ondas lentas de gran amplitud y baja frecuencia; los movimientos corporales son escasos, la respiración y la frecuencia cardiaca están disminuidas. Por otro lado, el sueño REM se divide a su vez en las fases N1, N2 y N3, en las cuales se incluye el sueño profundo. A lo largo de la noche, las diferentes etapas de sueño se alternan generando los ciclos de sueño, con frecuencia de cuatro a seis ciclos por noche con una duración de 90 a 120 minutos. .,11 $^{9}$

El TTS es el número de horas que duerme una persona y depende de la etapa de desarrollo en la que se encuentra el individuo. Diferentes estudios han reportado que las necesidades de TTS varían de acuerdo con la edad: de acuerdo con la National Sleep Fundation, un recién nacido ( 0 a 3 meses) requiere dormir entre 14 a 17 horas diarias, mientras que un adulto duerme entre siete a nueve horas y el adulto mayor entre siete y ocho horas por día. ${ }^{12} \mathrm{Sin}$ embargo, no existen reportes que sugieran un ajuste significativo en el TTS en un estado fisiológico particular, como el embarazo, pese a que esto implique un estado anabólico intenso.

\section{La melatonina y su relación con el sueño}

La melatonina es una indolamina ( $\mathrm{N}$-acetil-5-metoxitriptamina) producida principalmente por la glándula pineal. ${ }^{13,14}$ Adicionalmente, también es sintetizada por la retina, el timo, la médula ósea, el epitelio respiratorio, la piel, el intestino, las glándulas salivales, los linfocitos y las plaquetas.$^{15}$ Además, es producida en el ovario y en la placenta de la madre gestante, con un ritmo de secreción que impacta considerablemente en el progreso del embarazo. ${ }^{16}$

La secreción central de melatonina se produce como consecuencia de un mecanismo de control circadiano (patrón de secreción de 24 horas), sus niveles se mantienen disminuidos durante el día y aumentan a partir de las 20:00 horas, alcanzando un pico de secreción máxima desde las 3:00 horas, que se mantiene hasta poco antes de las 7:00 horas, luego de lo cual disminuyen abruptamente. ${ }^{17}$ Este pico máximo de secreción puede verse disminuido de forma total o parcial si el sujeto se expone a la luz durante la noche, debido a que la secreción está controlada por el núcleo supraquiasmático, cuyas funciones se encuentran sincronizadas por el ciclo de luz-oscuridad y, por tanto, funciona como un reloj endógeno regulador de diversos procesos..$^{18}$ La síntesis de melatonina es estimulada a través de la vía multisináptica retino-hipotalámico-pineal, integrada por la retina (punto de reconocimiento del estado lumínico), el núcleo paraventricular, el núcleo intermedio lateral y el ganglio superior cervical, que finalmente se comunica con la glándula pineal. ${ }^{17}$ Durante el día, los fotorreceptores (conos y bastones) de la retina se encuentran hiperpolarizados y en reposo, mientras que con la oscuridad se activan y estimulan la vía retino-hipotalámico-pineal, liberando noradrenalina en las terminaciones simpáticas que inervan la glándula pineal y estimulando la síntesis de melatonina en los pinealocitos. ${ }^{19}$ 


\section{El sueño en la mujer embarazada}

Los periodos fisiológicos normales a los que la mujer está sujeta a lo largo de la vida, como la pubertad, la menstruación, el embarazo y la menopausia, se asocian con el riesgo de padecer algún tipo de trastorno del sueño. ${ }^{20}$ Los cambios fisiológicos que ocurren durante el embarazo también se asocian con cambios en la arquitectura del sueño. EI TTS aumenta ligeramente en el primer trimestre del embarazo comparado con el estado no gestante ${ }^{21}$ y disminuye progresivamente durante el tercer trimestre. Las alteraciones del sueño están presentes en los tres tercios de la gestación, pero durante el tercer trimestre se observa un mayor impacto en la calidad y cantidad de sueño;, en este periodo, el TTS aumenta aproximadamente media hora y se acompaña de reducción en la calidad de sueño debido al síndrome de piernas inquietas y despertar nocturno, que se reportan con mayor frecuencia. ${ }^{20}$

Las alteraciones del sueño se han asociado con algunos cambios hormonales, entre ellos:

- Aumento de los niveles de progesterona, que causa fatiga y somnolencia diurna.

- Aumento de los niveles de estrógenos, que disminuye selectivamente la activación del sueño REM en el área preóptica ventrolateral.

- Alteraciones en la secreción de cortisol, que disminuye el sueño REM y aumenta el sueño de ondas lentas.

- Alteraciones de los niveles de prolactina, cuya secreción exacerba el sueño de ondas lentas.

- Alteraciones de los niveles de hormona del crecimiento, que se asocian con el inicio y mantenimiento del sueño de ondas lentas, además de desempeñar un papel importante en el crecimiento y desarrollo del feto. ${ }^{21,23}$

Si bien estos cambios obedecen a un proceso fisiológico normal en la mujer embarazada, pueden generar complicaciones, especialmente cuando se presenta obesidad, ya que la prevalencia de apnea del sueño es mayor. Además, en varios estudios se ha descrito que las mujeres con preeclampsia, padecimiento asociado con la obesidad materna, presentan más frecuentemente alteraciones respiratorias, eventos de hipoxia nocturna, apnea obstructiva del sueño $0^{24}$ y ronquidos. ${ }^{24,25}$

\section{Importancia de la melatonina en el embarazo}

Se sabe que los niveles séricos de melatonina en la mujer tienen una ritmicidad diurna con incrementos importantes hacia el final del embarazo. Las concentraciones de melatonina en la mujer gestante durante la fase de sueño (02:00 horas) presentan un ligero aumento a partir de la semana 24 de gestación, y alcanzan un pico máximo alrededor de la semana 32 a la 36, en comparación con las mujeres no embarazadas. Estos niveles retornan a la normalidad los dos días posteriores al parto. ${ }^{26}$

Las concentraciones de melatonina materna presentan una correlación significativa y estrecha con los niveles encontrados en la vena umbilical $(r=0.924, p<0.001)$. Además, se ha observado que los niveles de melatonina en sangre de cordón umbilical son más elevados en los neonatos nacidos durante la noche en comparación con aquellos que nacieron durante el día. Estos eventos sugieren que la melatonina es transferida de la sangre materna a la circulación fetal con alta eficiencia, a pesar de que el feto puede producir su propia melatonina con una ritmicidad circadiana. ${ }^{27}$

La información fotoperiódica percibida por la madre desde el ambiente externo, que promueve la secreción de melatonina, desempeña un papel importante en sincronizar el inicio de los procesos circadianos en el feto. ${ }^{16}$ De manera que durante la vida intrauterina, el organismo fetal "aprende" o programa sus mecanismos fisiológicos para determinar los ciclos de luz-oscuridad a través de la expresión de diversos genes reloj como Bmal-1, Per1-3, Cry1-2, en un efecto dependiente de los ritmos de secreción de melatonina materna. ${ }^{28}$

Además de los efectos sobre los patrones circadianos en el feto, se ha propuesto que la melatonina modula otros procesos fisiológicos, ${ }^{28}$ como los estados inflamatorio y antioxidante, la función pancreática, la motilidad intestinal, el peso corporal, la reproducción y el efecto anti jet lag.19

La melatonina también parece ser esencial para llevar a término un buen embarazo. De acuerdo con el reporte de Sandyk et al. (1992), esta hipótesis está basada en distintas observaciones:

- Los niveles de melatonina en plasma aumentan entre 200 y $300 \%$ en las primeras 20 semanas de embarazo.

- La melatonina disminuye la contracción uterina.

- La melatonina estimula la secreción de progesterona, la cual disminuye la contracción uterina y previene un rechazo inmunológico del trofoblasto, el cual tiene la función de aportar nutrimentos de la madre al feto a través de la arteria uterina. ${ }^{29}$ La alteración de dichos trofoblastos provoca restricción del crecimiento intrauterino y preeclamsia. ${ }^{22}$ 
- La melatonina inhibe la síntesis de prostaglandinas en el embarazo, las cuales son un importante inductor de la contracción uterina y la labor de parto.

- La pinealectomía incrementa el número de abortos espontáneos en ratas embarazadas.

\section{La melatonina y su relación con el bajo peso y el metabolismo del recién nacido}

El bajo peso al nacer es consecuencia de distintos factores estresantes (nutricionales, hormonales y aporte de oxígeno) a los que el feto es sometido durante la gestación, y ante los cuales presenta estrategias de adaptación bioquímicas, estructurales 0 funcionales que lo protegen de dicho estrés a costa de la ganancia de peso y la correcta maduración de órganos y sistemas, promoviendo la presencia de enfermedad metabólica en la edad adulta. Este fenómeno ha sido descrito por Barker como "hipótesis de origen fetal de las enfermedades metabólicas" 30 o por Lucas como "programación metabólica". ${ }^{31}$

El estudio del impacto que tiene el bajo peso al nacimiento ha sido relevante porque explica el aumento de la morbimortalidad neonatal e infantil, así como el aumento de obesidad y enfermedad metabólica; el análisis se ha ampliado a la programación intrauterina de diferentes tejidos, como la glándula tiroides, por la relevancia que tienen las hormonas tiroideas en la regulación del peso corporal y la ingestión de alimento. ${ }^{32}$

La disfunción placentaria, que se sugiere como consecuencia de la disminución en los niveles de melatonina o de las alteraciones en su ritmo de secreción, plantea la posibilidad de que la disrupción de los ciclos de luz-oscuridad durante la gestación tiene consecuencias metabólicas a largo plazo, entre ellos un incremento de la adiposidad, hiperleptinemia, hiperinsulinemia y menor tolerancia a la glucosa. ${ }^{33,34}$ Aunque los mecanismos a través de los cuales se generan estas alteraciones no han sido comprendidos en su totalidad, se ha sugerido que la melatonina puede desempeñar un papel importante en el establecimiento de las alteraciones metabólicas en la edad adulta. ${ }^{33}$

Las funciones de la melatonina materna podrían dividirse en las que pueden afectar las funciones de la placenta y las que impactan directamente al organismo fetal. ${ }^{16}$ Respecto a las primeras, se ha descrito que la melatonina contrarresta la generación de radicales libres evitando el estrés oxidativo que caracteriza las etapas iniciales de alteraciones de la placenta. En este sentido, las mujeres con preeclampsia que muestran niveles bajos de melatonina presentan deficiencia del paso de nutrimentos y del aporte de oxígeno al feto, lo que promueve complicaciones del embarazo, parto prematuro y bajo peso al nacimiento. ${ }^{16,35}$

Por otra parte, en función de su ritmo de secreción, la melatonina producida por el organismo materno es transportada fácilmente a través de la placenta y alcanza la circulación fetal. Es fundamental para regular algunas funciones durante el desarrollo: ${ }^{35}$ maduración del sistema nervioso, regulación de la temperatura neonatal, ${ }^{36}$ determinación de los ritmos circadianos de otros tejidos como el hígado, ${ }^{16}$ sincronización de los ciclos circadianos del feto, ${ }^{16,28,35}$ mediación en la expresión del gen de leptina en los adipocitos a través de su interacción con receptores tipo MT1 (melatonin receptors), ${ }^{37}$ lo que promueve el control de la ingestión de alimento y favorece la regulación del peso corporal; ${ }^{38,39}$ así como la función adecuada del páncreas y el metabolismo de la glucosa. ${ }^{40}$

Lo anterior resalta la relevancia del estudio de los problemas de sueño durante la gestación, para promover un estado homeostático en la madre que favorezca el adecuado crecimiento y desarrollo fetal, asegure el estado de salud del neonato y prevenga de forma temprana el desarrollo de obesidad y enfermedades metabólicas.

\section{Conclusiones}

La reducción en la cantidad de sueño o las alteraciones del sueño promueven cambios en la secreción circadiana de melatonina materna, que pueden repercutir en el aporte de esta hormona a la unidad feto-placentaria. El impacto negativo del bajo aporte de melatonina se ha descrito en relación con la estructura y función placentaria, que afecta el aporte de oxígeno y nutrimentos al feto; la afectación de la maduración del núcleo supraquiasmático fetal, que conlleva a la alteración de los ciclos circadianos; la resistencia a insulina y disfunción en la secreción de la leptina, alterando el control de la ingestión de alimento y la ganancia de peso corporal. Todos estos mecanismos pueden contribuir de alguna manera al bajo peso al nacimiento, generando un fenómeno de programación metabólica por alteración del sueño, que repercute en el desarrollo de obesidad y enfermedad metabólica en la edad adulta, por lo que es necesario promover estrategias de higiene del sueño durante este periodo que favorezcan la salud materna y del neonato para prevenir tempranamente el desarrollo de obesidad y enfermedad metabólica. 


\section{Bibliografía}

1. Carneiro G, Zanella MT. Obesity metabolic and hormonal disorders associated with obstructive sleep apnea and their impact on the risk of cardiovascular events. Metabolism.2018;84:76-84.

2. Touitou YV, Reinberg A, Touitou D. Association between light at night, melatonin secretion, sleep deprivation, and the internal clock: health impacts and mechanisms of circadian disruption. Life Sci. 2017;173:94-106

3. Malone S, Zemel B, Compher C, Souders M, Chittams J, Thompson A et al. Social jet lag, chronitype and body mass index in 14-17-year-old adolescents. Chronobiol Int. 2016;11:1-12.

4. Albrecht U, Ripperger JA. Circadian clocks and sleep: impact of rhythmic metabolism and waste clearance on the brain. Trends Neurosci. 2018:41:677-688.

5. Reiter RJ, Tan DX, Korkmaz A, Ma S. Obesity and metabolic syndrome: association with chronodisruption, sleep deprivation, and melatonin suppression. Ann Med. 2012;44:564-577.

6. Carrillo-Mora P, Ramírez-Peris J, Magaña-Vázquez K. Neurobiología del sueño y su importancia: antología para el estudiante universitario. Rev Fac Med (Mex). 2013;56:5-15.

7. van Der Zwan JE, De Vente W, Tolvanen M, Karlsson H, Buil JM, Koot HM, et al. Longitudinal associations between sleep and anxiety during pregnancy, and the moderating effect of resilience, using parallel process latent growth curve models. Sleep Med. 2017;40:63-68.

8. Nodine PM, Matthews EE. Common sleep disorders: management strategies and pregnancy outcomes. J Midwifery Womens Health. 2013; 58:368-377.

9. Carley DW, Farabi SS. Physiology of sleep. Diabetes Spectr. 2016;29:5-9.

10. Tain YL, Huang LT, Hsu CN. Developmental programming of adult disease: reprogramming by melatonin? Int J Mol Sci. 2017;18:426.

11. Patel AK, Araujo JF. Physiology, sleep stages. EE. UU.: StatPearls [sitio web]; 2018.

12. Hirshkowitz M, Whiton K, Albert SM, Alessi C, Bruni O, DonCarlos L, et al. National Sleep Foundation's updated sleep duration recommendations: final report. Sleep Health. 2015;1:233-243.

13. Pfeffer M, Korf HW, Wicht $H$. Synchronizing effects of melatonin on diurnal and circadian rhythms. Gen Comp Endocrinol. 2018;258:215-221.

14. Pin Arboledas G, Morell Salort M, Mompo Marabotto L. Higiene de sueño y melatonina. En: AEPap. Curso de Actualización Pediatría. España: Exlibris; 2014.

15. Singh M, Jadhav HR. Melatonin: functions and ligands. Drug Discov Today. 2014;19:1410-1418.

16. Reiter RJ, Tan DX, Korkmaz A, Rosales-Corral SA. Melatonin and stable circadian rhythms optimize maternal, placental, and fetal physiology. Hum Reprod Update. 2014;20:293-307

17. Guerrero JM, Carrillo-Vico A, Lardone PJ. La melatonina. Invest Cienc. 2007;373:32-38

18. Reiter RJ, Rosales-Corral S, Coto-Montes A, Boga JA, Tan DX, Davis $\mathrm{JM}$, et al. The photoperiod, circadian regulation and chronodisruption: the requisite interplay between the suprachiasmatic nuclei and the pineal and gut melatonin. J Physiol Pharmacol. 2011;62:269-274.

19. Carpentieri A, Díaz-De Barboza G, Areco V, Peralta-López M, Tolosa-De Talamoni N. New perspectives in metlatonin uses. Phamacol Res. 2012;65:437-444

20. Tamanna S, Geraci SA. Major sleep disorders among women: (women's health series). South Med J. 2013:106:470-478.

21. Balserak BI, Lee K. Sleep disturbances and related disorders in pregnancy. En: Balserak BI, Lee K, editores. Principles and practice of sleep medicine. EE. UU.: Saunders; 2010.
22. Soliman A, Lacasse A, Lanoix D, Sagrillo-Fangundes L, Boulard V, Vaillancour C. Placental melatonin system is present throughout pregnancy and regulates villous trophoblast differentiation. J Pineal Res. 2015;59:38-46.

23. Parra O, Sánchez-Armengol A, Capote F, Bonnin M, Arboix A, Campos-Rodríguez $\mathrm{F}$, et al. Efficacy of continuous positive airway pressure treatment on 5-year survival in patients with ischaemic stroke and obstructive sleep apnea: a randomized controlled trial. J Sleep Res. 2015;24:47-53.

24. Pien GW, Pack AI, Jackson N, Maislin G, Macones GA, Schwab RJ. Risk factors for sleep-disordered breathing in pregnancy. Thorax. 2014; 69:371-377.

25. Domínguez JE, Street L, Louis J. Management of obstructive sleep apnea in pregnancy. Obstet Gynecol Clin North Am. 2018;45:233-247.

26. Nakamura $Y$, Tamura $H$, Kashida S, Takayama H, Yamagata $Y$, Karube $A$, et al. Changes of serum melatonin level and its relationship to feto-placental unit during pregnancy. J Pineal Res. 2001;30:29-33.

27. Okatani Y, Okamoto K, Hayashi K, Wakatsuki A, Tamura S, Sagara Y. Maternal-fetal transfer of melatonin in pregnant women near term. J Pineal Res. 1998;25:129-134.

28. Chen YC, Sheen JM, Tiao MM, Tain YL, Huang LT. Roles of melatoin in fetal programming in compromised pregnancies. Int J Mol Sci. 2013; 14:5380-5401.

29. Sandyk R, Anastasiadis PG, Anninos PA, Tsagas N. The pineal gland and spontaneous abortions: implications for therapy with melatonin and magnetic field. Int J Neurosci. 1992;62:243-250.

30. Barker DJ. The fetal and infant origins of adult disease. BMJ. 1990; $301: 1111$.

31. Lucas A. Programming by early nutrition in man. Ciba Found Symp. 1991;156:38-50.

32. Ayala-Moreno R, Racotta R, Anguiano B, Aceves C, Quevedo L. Perinatal undernutrition programmes thyroid function in the adult rat offspring. Br J Nutr. 2014;10:2207-2215.

33. Méndez N, Halabi D, Spichiger C, Salazar ER, Vergara K, Alonso-Vásquez $\mathrm{P}$, et al. Gestational chronodisruption impairs circadian physiology in rat male offspring, increasing the risk of chronic disease. Endocrinology. 2016;157:4654-4668.

34. Zhang $\mathrm{Y}$, Sun $\mathrm{CM}, \mathrm{Hu} X \mathrm{X}$, Zhao $\mathrm{Y}$. Relationship between melatonin receptor $1 \mathrm{~B}$ and insulin receptor substrate 1 polymorphisms with gestational diabetes mellitus: a systematic review and meta-analysis. Sci Rep. 2014;4:6113.

35. Reiter RJ, Tan DX, Tamura H, Cruz MH, Fuentes-Broto L. Clinical relevance of melatonin in ovarian and placental physiology: a review. Gynecol Endocrinol. 2014;30:83-89.

36. Seron-Ferre M, Reynolds $H$, Méndez NA, Mondaca M, Valenzuela F, Ebensperger $\mathrm{R}$, et al. Impact of maternal melatonin suppression on amount and functionality of brown adipose aissue (BAT) in the newborn sheep. Front Endocrinol (Lausanne). 2015;5:232.

37. Zlotos DP, Jockers R, Cecon, E, Rivara S, Witt-Enderby PA. MT1 and MT2 melatonin receptors: ligands, models, oligomers, and therapeutic potential. J Med Chem. 2014;5:3161-3185.

38. Markwald RR, Melanson EL, Smith MR, Higgins J, Perreault L, Eckel RH, et al. Impact of insufficient sleep on total daily energy expenditure, food intake, and weight gain. Proc Natl Acad Sci U S A. 2013;110:5695-5700.

39. Terrón MP, Delgado-Adámez J, Pariente JA, Barriga C, Paredes SD, Rodríguez AB. Melatonin reduces body weight gain and increases nocturnal activity in male Wistar rats. Physiol Behav. 2013;118:8-13.

40. Onaolapo AY, Onaolapo OJ. Circadian dysrhythmia-linked diabetes mellitus: examining melatonin's roles in prophylaxis and management. World J Diabetes. 2018;9:99-14. 